\title{
CAD/CAM FABRICATION OF A PATIENT-SPECIFIC PEEK IMPLANT FOR MARGINAL MANDIBULAR RECONSTRUCTION: A TECHNICAL REPORT
}

\author{
Mohamed Moustafa Said*, Mohamed Shady*, Mohamed Elmetwally**, \\ Marco Youssef ${ }^{* * *}$ and Salah Abdelftah Hegazy ${ }^{* * * *}$
}

\begin{abstract}
Surgical reconstruction of mandibular defects is usually considered a challenge. This technique describes a workflow for CAD/CAM fabrication and insertion of PEEK implant for partial marginal resection of the mandibular angle bone for a 17-year-old young female diagnosed with low grade osteosarcoma. High resolution cone beam computed tomography scan was performed for digital planning. 3D rendering based on the DICOM data was performed using Ceramill Mind - software (Amann Girrbach AG), which produced a three-dimensional model of the mandible. The implant was first 3D designed on computer (Ceramill Mind - software), milled into wax and then finally produced using PEEK injection molding technique. The patients' aesthetics and functions regarding speech, mastication, and normal opening without deviation were maintained as detected after 6-month follow-up. Combination between CAD/CAM and use of PEEK material in the reconstruction of mandibular defects could be a promising treatment option to restore the physiologic functions of the mandible.
\end{abstract}

KEYWORDS: 3D, Bone graft, CAD/CAM, PEEK

\section{INTRODUCTION}

Osteosarcoma is a bone cancer that develops from osteoblast cells that from the bone. It is the most common type of cancer that occurs in bone. Despite it can develop at any age, it mainly affects children, teen and young adults. Osteosarcomas can be classified as high grade, intermediate grade, or low grade, depending on how likely the tumour will grow and spread.

Resection in the mandible can lead to either marginal or segmental mandibulectomy defects. Surgical resection can have significant negative effects in patients' quality of life because it could deteriorate the basic functions such as mastication, aesthetics and speech. ${ }^{1,2}$ Surgical reconstruction of

* Lecturer, Prosthodontic Department, Faculty of Dentistry, Mansoura University, Egypt

** Assistant Professor of Surgical Oncology, Faculty of Medicine, Mansoura University, Egypt

*** Resident of Surgical Oncology, Faculty of Medicine, Mansoura University, Egypt

**** Professor, Prosthodontic Department, Faculty of Dentistry, Mansoura University, Egypt 
mandibular defects is usually a challenge to both the oncologist and the prosthodontist as the anatomy of the mandibular ridge is not similar to other bones and the nature of temporomandibular joint is different from other joints. ${ }^{3}$

Marginal mandibulectomy defects involve the resection of a single cortex of the mandible and can be restored in different ways, such as osseocutaneous microvascular free flaps or non-vascularized bone grafts. Numerous autogenous and alloplastic material have been used in maxillofacial reconstruction. A potential candidate is polyetheretherketone (PEEK). PEEK is a semi-crystalline polyaromatic linear polymer that exhibits an excellent combination of strength, stiffness, durability, and environmental resistance. It is also highly resistant to degradation in a biological environment making it an excellent choice as an implantable medical implant. ${ }^{4}$

Advances in computer-aided design and manufacturing (CAD/CAM) technology have created an increasing number of applications for virtual surgical planning in oro-maxillo-facial surgery., 5 This technique describes a workflow for CAD/CAM fabrication and insertion of PEEK substitute for partial marginal resection of the mandibular bone for a 17-year-old young female diagnosed with low grade osteosarcoma.

\section{Technique Presentation}

A 17-year-old female patient was referred to

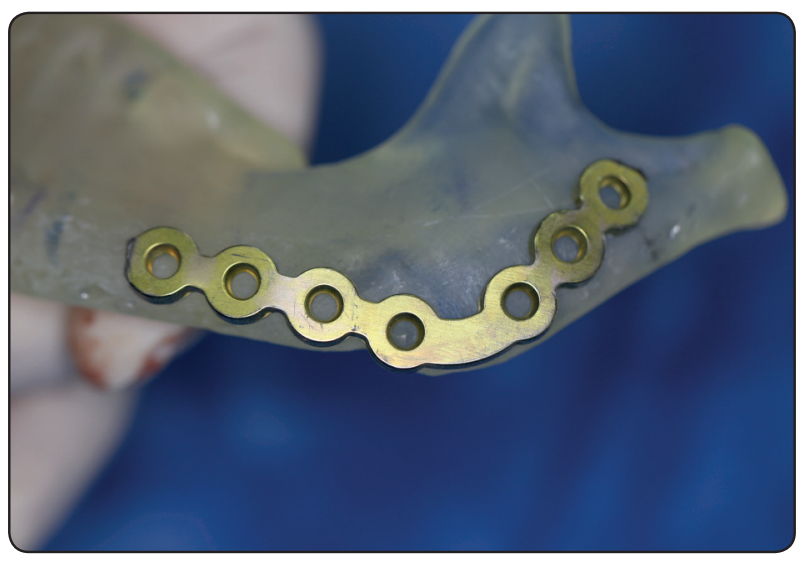

Fig. (1) Three-dimensional model of the mandible used for planning and adaptation of surgical titanium plate the Department of Prosthodontics by Oncology Centre, Mansoura University reporting low grade osteosarcoma in the inferior border of the mandible. High resolution cone beam computed tomography scan was performed for digital planning. 3D rendering based on the DICOM data was performed using Ceramill Mind - software (Amann Girrbach AG), which produced a three-dimensional model of the mandible (Fig 1). After consultation with the Oncology Centre about tumor resection site and size, a patient-specific PEEK implant (ceramill ${ }^{\circledR}$ peek) was produced virtually using the mirroring technique. The implant was first 3D designed on computer (Ceramill Mind - software), milled into wax (Fig 2) and then finally produced using PEEK injection molding technique.

Under general anaesthesia, an extended submandibular approach was performed and the bone was exposed after exposure of the facial nerve branches. The lesion was outlined and resected using surgical guide (Fig 3). The preformed PEEK implant was then adapted to the defect with a slight minimal correction to completely and passively fit. The implant was fixed to the bone using titanium plate and screws (Fig 4). The post-operative period was uneventful and the patient was discharged on the third day with antibiotic therapy (Fig 5 and 6). The patients' aesthetics and functions regarding speech, mastication, and normal opening without deviation were maintained as detected during 6-month follow-up.

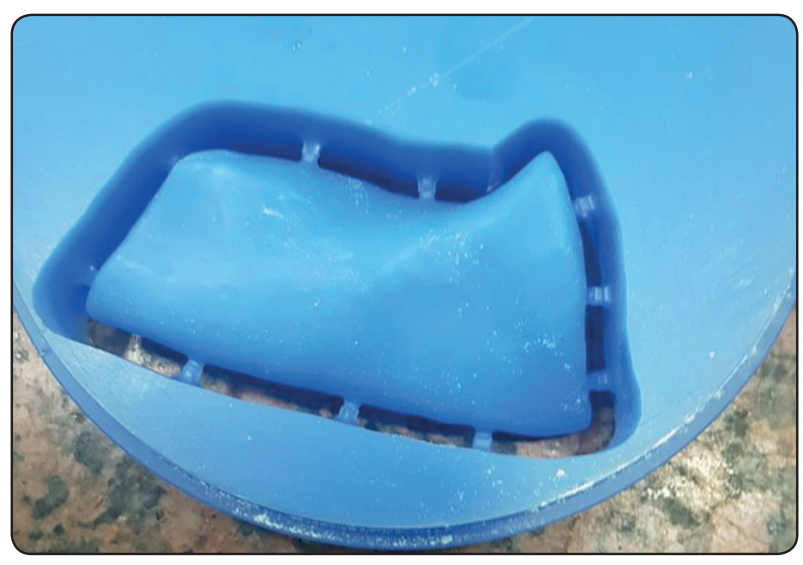

Fig. (2) PEEK implant milled into wax after 3D designing using the mirroring technique 


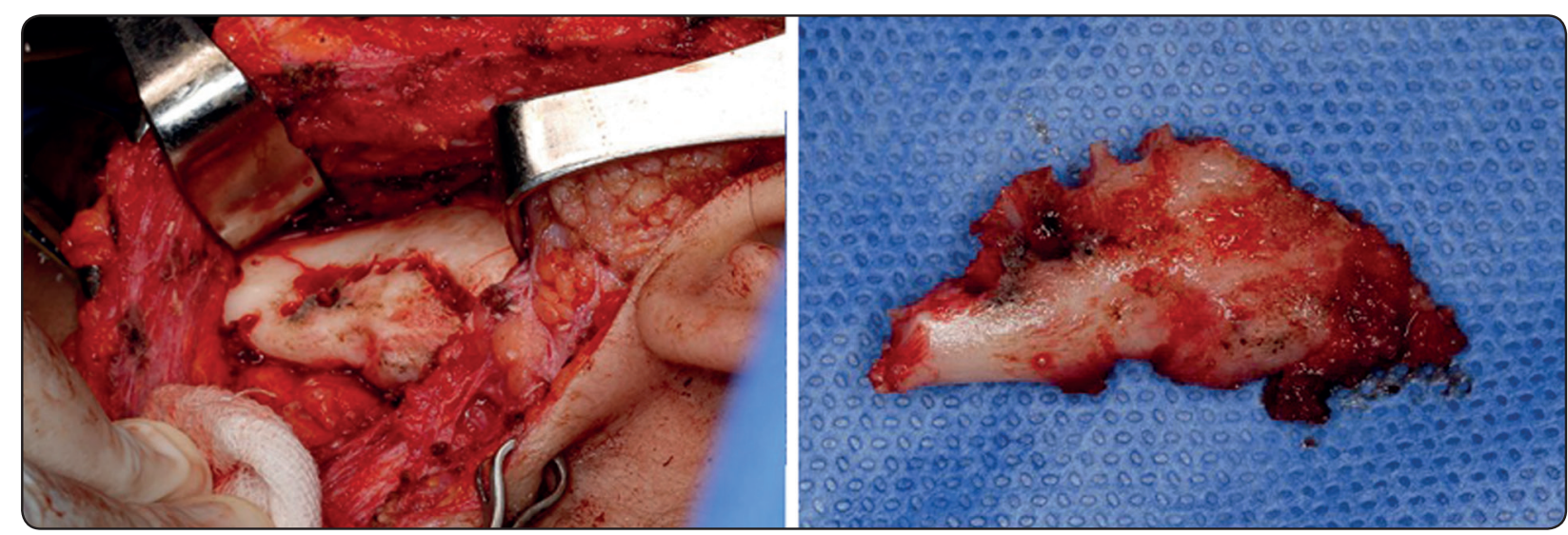

Fig. (3) The lesion was outlined and resected using surgical guide

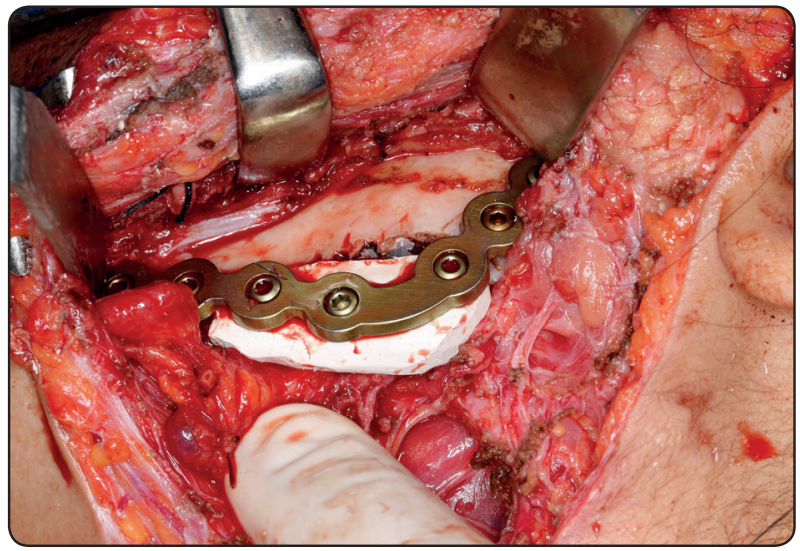

Fig. (4) The implant was fixed to the bone using titanium plate and screws with a slight minimal correction to completely and passively fit

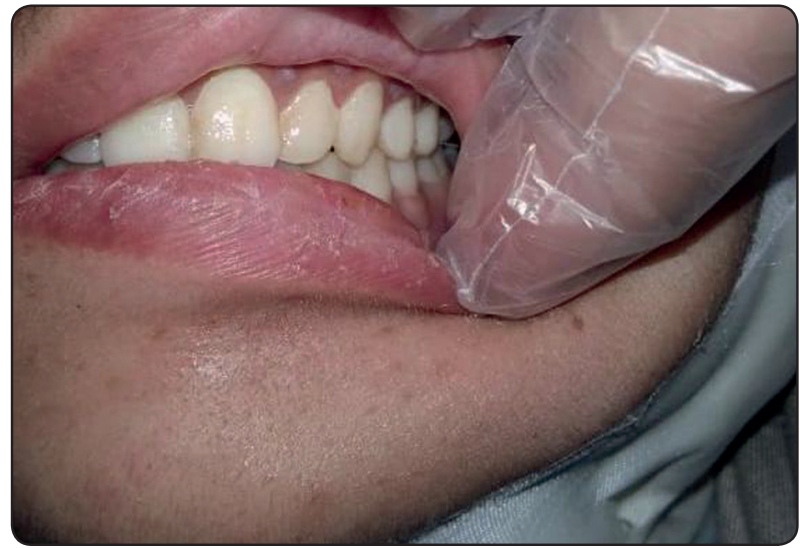

Fig. (5) The post-operative clinical view showing the patient' normal occlusion after the surgical reconstruction

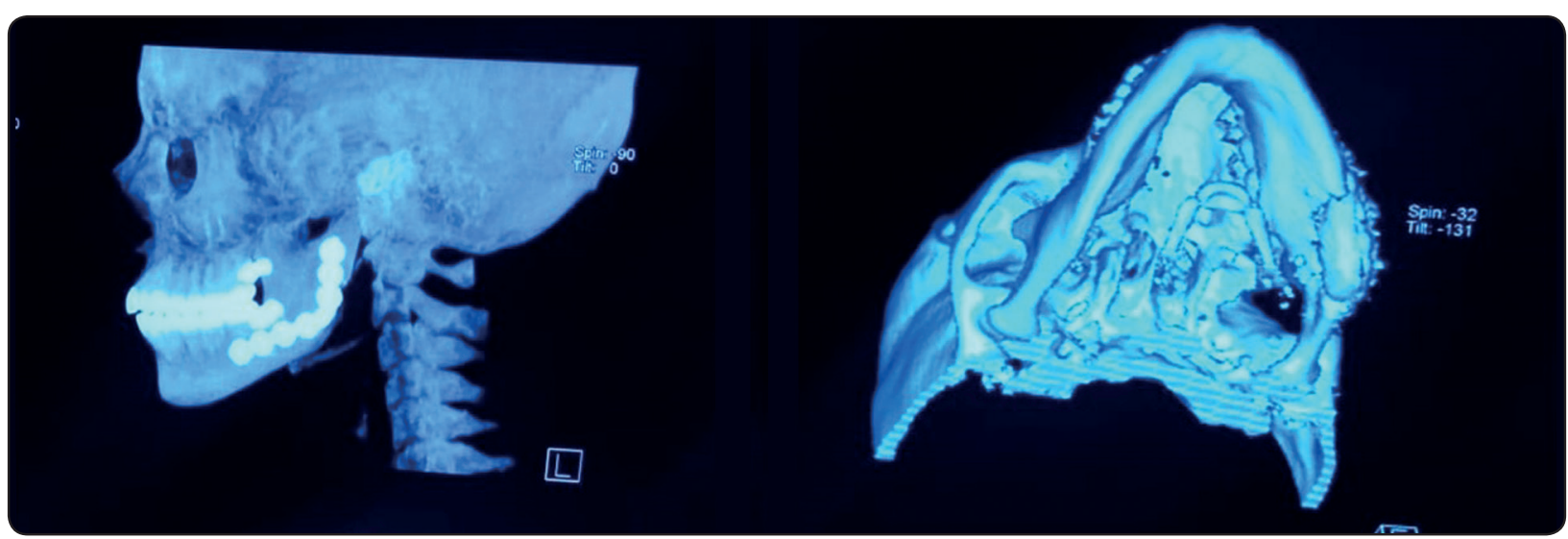

Fig. (6) The post-operative X-ray showing the PEEK implant, titanium plate and screws 


\section{DISCUSSION}

Most commonly, autogenous bone grafts, free tissue transfer, methylmethacrylate, or different types of bone cement are used for reconstructing craniofacial defects. However, all have associated with disadvantages. Autogenous bone grafts and free tissue transfer (eg, fibula osteocutaneous free flaps) exhibit resorption and donor site morbidity. ${ }^{7}$ Methylmethacrylate can elicit foreign body reactions, resulting in high infection and extrusion rates. Bone cements such as carbonated calcium phosphate paste and hydroxyapatite cement exhibit poor workability and need to be sculpted during surgery to achieve a satisfactory aesthetic result.

A custom-made PEEK implant was fabricated using injection molding technique. This technique is less costly than subtractive type and allows for reconstruction of large defects. Computerdesigned alloplastic PEEK implants might represent a milestone in the evolution of planning and realization of $3 \mathrm{D}$ surgical reconstructions. It is considered to be an advanced biomaterial used in medical implants engineered for strength, stability and biocompatibility. In this report, the patients' functions regarding speech, mastication, normal opening without deviation were maintained as detected during follow-up. The patient's aesthetics regarding mandibular symmetry was also maintained. This surgical reconstruction has also provided a support to the bone to prevent any future unexpected mandibular fracture. However, long term follow-up is required to back up by evidence the effectiveness of the employed protocol.

\section{CONCLUSION}

Combination between CAD/CAM and use of PEEK material in reconstruction of mandibular defects could be a promising treatment option to restore the physiologic functions of the mandible.

\section{REFERENCES}

1. Warshavsky A, Fliss DM, Frenkel G, Kupershmidt A, Moav N, Rosen R, Sechter M, Shapira U, Abu-Ghanem S, Yehuda M, Zaretski A, Yanko-Arzi R, Reiser V, Horowitz G. Quality of life after mandibulectomy: the impact of the resected subsite. Int J Oral Maxillofac Surg. 2019 Oct;48(10):1273-1278.

2. Otomaru T, Sumita YI, Chang Q, Fueki K, Igarashi Y, Taniguchi $\mathrm{H}$. Investigation of predictors affecting food mixing ability in mandibulectomy and/or glossectomy patients. J Prosthodont Res. 2009 Jul;53(3):111-5.

3. Chang CL, Wang DH, Yang MC, Hsu WE, Hsu ML. Functional disorders of the temporomandibular joints: Internal derangement of the temporomandibular joint. Kaohsiung J Med Sci 2018;34:223-230.

4. Oh JH. Recent advances in the reconstruction of craniomaxillofacial defects using computer-aided design/computer-aided manufacturing. Maxillofac Plast Reconstr Surg 2018 5;40:2.

5. Berrone M, Aldiano C, Pentenero M, Berrone S. Correction of a mandibular asymmetry after fibula reconstruction using a custom-made polyetheretherketone (PEEK) onlay after implant supported occlusal rehabilitation. Acta Otorhinolaryngol Ital 2015;35:285-288.

6. Chang EI, Boukovalas S, Liu J, Largo RD, Hanasono MM, Garvey PB. Reconstruction of Posterior Mandibulectomy Defects in the Modern Era of Virtual Planning and Three-Dimensional Modeling. Plast Reconstr Surg. 2019 Sep;144(3):453e-462e.

7. Ling XF, Peng X, Samman N. Donor-site morbidity of free fibula and DCIA flaps. J Oral Maxillofac Surg 2013;71:1604-1612. 